\title{
\begin{tabular}{l|l} 
POLITIQUES \& & Politiques et management public
\end{tabular}

\section{Les démarches de contractualisation comme processus de conception : l'émergence du contrôle de gestion médicalisé à l'hôpital}

How contracting process contributes to the emergence of new medico-economic monitoring and control?

\section{Sophie Colasse et Michel Nakhla}

\section{OpenEdition \\ Journals}

Édition électronique

URL : http://journals.openedition.org/pmp/4537

ISSN : 2119-4831

Éditeur

Institut de Management Public (IDPM)

\section{Édition imprimée}

Date de publication : 15 septembre 2011

ISSN : 0758-1726

Référence électronique

Sophie Colasse et Michel Nakhla, «Les démarches de contractualisation comme processus de conception : l'émergence du contrôle de gestion médicalisé à l'hôpital », Politiques et management public [En ligne], Vol 28/3 | 2011, mis en ligne le 04 novembre 2013, consulté le 19 avril 2019. URL : http://journals.openedition.org/pmp/4537 


\title{
Les démarches de contractualisation comme processus de conception : l'émergence 0 du contrôle de gestion médicalisé à l’hôpital
}

\author{
Sophie Colasse et Michel Nakhla* \\ Centre de Gestion Scientifique, École des Mines ParisTech \\ 60, Bd Saint Michel , 75272 Paris cedex 06.
}

\section{Résumé}

À l'hôpital, le contrôle de gestion a longtemps été utilisé comme outil de contrôle réglementaire au service de la tutelle ou comme outil au service des politiques de santé. Plus généralement, il a souffert d'un certain nombre d'écueils parmi lesquels une insuffisante prise en compte de la dimension interne et organisationnelle qui s'explique en partie par une vision réduite du contrôle de gestion tournée vers la simple connaissance des coûts. L'objet de ce papier consiste à étudier les conditions d'émergence de nouveaux concepts de contrôle de gestion à l'hôpital. Le recours à la théorie $C-K$, nous semble être un cadre d'analyse pour discuter l'hypothèse selon laquelle, les démarches de contractualisation sont des processus de conception. À partir de ce cadre théorique et de l'analyse d'expériences contractuelles à l'hôpital, nous faisons l'hypothèse que le renforcement des démarches de contractualisation contribue à l'émergence de nouvelles démarches de contrôle de gestion à l'hôpital, au sens large d'un pilotage médico-économique.

(c) 2011 IDMP/Lavoisier SAS. Tous droits réservés

Mots clés : Conception, contrôle de gestion médicalisé, contractualisation

\section{Abstract}

How contracting process contributes to the emergence of new medico-economic monitoring and control? Financial reporting has long been used as a tool of regulatory control by the supervisory authority or employed in health policies concerning hospitals. More generally, a number of problems over financial reporting have come to light including an inadequate analysis of the internal organization of hospitals which can be partially

*Auteur correspondant : Nakhla@ensmp.fr doi:10.3166/pmp.28.311-331 @ 2011 IDMP/Lavoisier SAS. Tous droits réservés 
explained by a vision limited to costs. The aim of this paper is to study the conditions of the emergence of new concepts of control in hospital management. It will argue that the C-K theory provides an analytical framework for understanding the contract process as a design process. This theoretical framework along with the analysis of examples of contracting reveals that the contracting process contributes to the emergence of new representations of control in the form of medico-economic monitoring.

(c) 2011 IDMP/Lavoisier SAS. Tous droits réservés

Keywords: Design, medico-economic monitoring, contract.

\section{Introduction}

Les organisations publiques connaissent depuis quelques années des évolutions profondes : introduction de démarches gestionnaires, évaluation de l'activité, contractualisation des objectifs... Ces évolutions remettent en cause, dans bon nombre de situations, la nature des échanges au sein de ces organisations. Ainsi, par exemple, les exigences de performance, imposées à certains services, entraînent une reconfiguration des relations entre l'entreprise publique, ses tutelles et ses usagers (nouveaux principes de tarification, émergence ou transformation des politiques contractuelles,...).

Le secteur hospitalier français est à cet égard révélateur. Bien que jouissant d'une bonne réputation, il connaît depuis plusieurs années des difficultés budgétaires importantes qui ont conduit à l'introduction régulière d'outils de gestion (tarification à l'activité, logiques de contrôle de gestion, tableaux de bords médico-économiques, principe de la convergence des tarifs entre public et privé...).

De l'avis même des acteurs, la greffe des outils de gestion n'a pas vraiment pris dans l'ensemble du système hospitalier et s'explique par les rapports ambigus entre l'État et l'hôpital public en France. Ils sont faits d'un mélange entre une régulation bureaucratique (ou professionnelle), une régulation par le marché et une régulation politique : la tutelle exerce un contrôle bureaucratique sur les hôpitaux qui sont en concurrence entre eux et avec les systèmes privés (Mintzberg, 1979).

Dans ce contexte, la mise en place du contrôle de gestion à l'hôpital constitue un outil de gestion qui peine à trouver sa légitimité. Dès les années 1970, Gauthier et al. (1975) expliquent que la logique de la profession médicale tend à rejeter toute approche quantitative, chiffrée des divers domaines de l'activité hospitalière. Aujourd'hui, si la mesure chiffrée est davantage répandue au sein de l'hôpital, c'est l'usage qui en est fait qui est parfois dénoncé à travers la recherche de performance et de rentabilité inhérente à la mise en place de la tarification à l'activité (T2A) par exemple (Expert, 2006; Escaffre, 2008). La fonction Contrôle de gestion a-t-elle sa place dans une organisation telle que l'hôpital qui, rappelons-le, est définie par ses missions; selon le Code de la Santé Publique, le service public hospitalier a obligation d'assurer ou de concourir à la prévention, l'enseignement universitaire, la recherche, la qualité des soins et la sécurité. Il n'est donc jamais fait référence à un objectif de profit via un développement de l'activité par exemple.

Pour autant, le droit lui-même pose la question de la conciliation entre intérêts médicaux et intérêts économiques; l'article R 4127-92 du Code de la Santé Publique commenté 
par le Conseil National de l'Ordre des Médecins rappelle que « le médecin doit prendre conscience des réalités économiques aussi bien dans la gestion d'un cabinet libéral que dans son exercice au sein d'un établissement public hospitalier ou privé. " [...] Tout médecin doit donc s'efforcer de trouver une adéquation entre le souci légitime de bien traiter ses patients et les réalités économiques ».

Selon plusieurs auteurs le contrôle peut être caractérisé en fonction de deux aspects, l'instrumentation comptable dans une perspective décisionnaire ou stratégique et le pilotage de la performance et de l'organisation (Gibert, 1980 ; Colasse, 2008). Si certains auteurs ont fait porter leur recherche sur la seule conception et le développement des systèmes de production de l'information comptable et son utilisation par les gestionnaires dans une perspective stratégique (Kaplan, 2003), d'autres auteurs partent du constat d'une focalisation du contrôle de gestion sur les seuls aspects instrumentaux particulièrement dans le cadre de l'introduction de la méthode $\mathrm{ABC}$, la chaîne liant données, décision et action doit finalement être renversée et c'est l'action qui doit donner du sens aux informations médico-économiques en leur conférant une finalité et une signification. Mévellec (2004) dénonce ainsi la non-adéquation entre données fournies et système d'actions. Le contrôle de gestion doit alors prendre une dimension se rapprochant du pilotage de la performance défini comme étant une « démarche qui s'attache à relier en permanence stratégie, action opérationnelle et mesure des performances » (Demeestere, Lorino et Mottis, 2003).

À travers ces travaux, l'instrumentation économique à l'hôpital est simultanément un moyen d'action et d'intervention dans l'organisation (Moisdon, 1998) et de mise en œuvre d'une stratégie d'établissement (Alvarez, 2000 ; Bouinot, 2005). Le diagnostic obtenu à partir de ces recherches sur l'hôpital peut être résumé de la façon suivante :

- Les hôpitaux ont tenté d'introduire un certain nombre d'outils de gestion. Ces outils ont été développés dans le cadre d'expérimentations qui restent trop isolées et traduisent les difficultés de l'hôpital à capitaliser les enseignements qui en sont issus et à prendre en compte la dimension interne.

- L'usage des outils du contrôle de gestion reste souvent restreint à la vérification au détriment d'un véritable pilotage de l'activité de l'hôpital.

- Le contrôle de gestion n'a été rendu possible, dans certains hôpitaux, que par la mise à disposition des gestionnaires hospitaliers d'outils de régulation externe : le Programme de Médicalisation des Systèmes d'Information ou de contractualisation interne. Ces outils présentent notamment l'avantage de croiser deux logiques, l'une économique et l'autre médicale.

- La mise en œuvre d'un nouveau mode de financement des hôpitaux en France sur la base d'une tarification à l'Activité (T2A) redonne, a priori, du sens aux outils du contrôle de gestion. Ce type de tarification présente l'intérêt d'inciter notamment les hôpitaux à minimiser les coûts. En effet, ils ne seront pas remboursés pour des dépenses dépassant le forfait correspondant au cas traité. À l'inverse, ils doivent pouvoir s'approprier les surplus découlant d'un séjour moins coûteux que le forfait.

- La plupart des outils du contrôle de gestion restent toutefois cantonnés à une seule logique budgétaire, celle de l'allocation des ressources en lien avec des préoccupations financières liées à la $\mathrm{T} 2 \mathrm{~A}$, sans impact sur les comportements organisationnels. Il en résulte un gap entre contrôle de gestion et organisation. 
Pour autant, la nouvelle gouvernance, introduite par l'ordonnance du 2 mai $2005^{1}$, associée à des démarches de contractualisation a tenté de réduire ce gap à travers un double objectif :

- d'une part, faciliter l'élaboration de projets médicaux visant à valoriser au mieux l'activité, et donc contribuer à réduire le déficit budgétaire;

- d'autre part, permettre l'élaboration de tableaux de bords « explicatifs » de la performance et différencier les secteurs par rapport à la performance globale de l'hôpital, grâce à une remontée d'informations sur les coûts, rendue possible par une comptabilité analytique.

L'évolution de la régulation du système hospitalier public en France est fondée sur un double mouvement : accroissement des moyens de contrôle externe de la production de soins, incitation à la responsabilisation des hôpitaux par une régulation contractuelle. Or ce processus externe, est décliné en interne sous la forme d'une contractualisation interne.

Dans ce cadre, l'objet de cet article est d'examiner l'émergence du contrôle de gestion médicalisé et le processus de contractualisation dont il est issu à partir d'une. Elle fournit, en effet, des éléments intéressants pour analyser les conditions et les difficultés d'émergence d'un contrôle de gestion médicalisé. Par ailleurs elle apporte, d'une façon plus générale, des éléments sur le processus de conception de nouveaux outils de gestion dans un contexte d'abondance d'informations économiques et médicales.

Notre hypothèse est que le concept de contrôle de gestion médicalisé amène aujourd'hui à poser la question non plus du contrôle mais de l'organisation, de la production et de l'utilisation collective des connaissances médico-économiques. Cette vision du contrôle de gestion repose sur de nouvelles démarches de contractualisation qui, favorisant les cycles d'apprentissages collectifs et permet la régénération simultanée d'outils de gestion et de nouvelles connaissances. Pour autant, la contractualisation interne ne saurait prendre la forme d'une déclinaison imposée des connaissances externes, d'objectifs de rationnement des ressources, sans fondement médical. Elle nécessite l'invention de nouvelles pratiques de gestion.

C'est à partir de l'expérience issue d'un projet de contractualisation interne mis en œuvre au sein d'un établissement public que nous esquisserons les caractéristiques de ces nouvelles pratiques en vue de répondre à un certain nombre de questions : dans quelle mesure contribue-t-elle au processus de contrôle de gestion médicalisé et partagé ? Quel est le statut du contrat ? Ces projets de contractualisation - déconcentration permettentelles de réduire le gap observé entre gestion et organisation sur la base de l'apprentissage de nouveaux rôles?

Notre démonstration s'articule en deux temps; après avoir montré que le concept de contrôle de gestion médicalisé et partagé s'est heurté à un certain nombre d'obstacles qui ont ralenti son développement au sein de l'organisation hôpital, nous étudions dans un second temps les fondements d'une démarche de contractualisation interne en tant que processus favorable à l'agrégation des connaissances et à l'émergence d'un concept nouveau, celui d'un contrôle de gestion médicalisé et partagé.

\footnotetext{
${ }^{1}$ La Nouvelle Gouvernance a été introduite dans le cadre du plan Hôpital 2007, devenu hôpital 2012 ; elle vise à responsabiliser le personnel soignant et non soignant, élargir l'autonomie de l'établissement et instaurer des contrats entre les différents acteurs de la santé. Concrètement il s'agit de mettre en place des pôles d'activité, en tant que regroupements de services hospitaliers, auxquels est attribuée une délégation de gestion (gestion du personnel, enveloppes d'investissement, contractualisation d'objectifs...). Ainsi, il s'agit bien de décloisonner l'hôpital en rapprochant les logiques médicales et administratives, à responsabiliser les différents acteurs, et à rapprocher la gestion du terrain.
} 


\section{L'émergence de la notion de contractualisation interne en réponse aux dysfonctionnements du contrôle de gestion à l'hôpital}

D'un point de vue théorique, l'émergence d'un véritable pilotage de la performance constitue une question très débattue et témoigne de l'évolution du concept de contrôle de gestion d'une logique de contrôle à une logique plus large de pilotage. Ces travaux attestent ainsi du caractère ambigu des rapports entre l'État et l'hôpital public autrement dit entre une régulation bureaucratique d'un côté et une régulation par le marché qui pourrait émerger de l'autre. Les débats qui en résultent se focalisent autour de trois enjeux :

(i) Le contrôle de gestion comme élément clé de la construction de la stratégie à l'hôpital. Plusieurs auteurs soulignent la dimension stratégique qui caractérise le contrôle de gestion à travers l'usage fait des données chiffrées obtenues par ce processus. Anthony (1988) a ainsi formalisé le lien entre contrôle et stratégie en définissant le contrôle de gestion comme un " processus par lequel les dirigeants influencent d'autres membres de l'organisation pour mettre en ceuvre les stratégies ». Dans ce contexte, l'hypothèse formulée par Bouquin (1997) s'applique; face au contrôle de gestion réactif, "introverti », de surveillance il existerait un contrôle de gestion proactif, extraverti qui est une partie clé du processus d'émergence et de construction de la stratégie de l'hôpital. L'ensemble de ces éléments illustrent donc bien le caractère stratégique du contrôle de gestion qui apparaît comme un système planifié d'aide au pilotage. Mais, si le contrôle de gestion apparaît comme étant un élément clé de la stratégie, il doit dès lors s'appuyer sur des outils maitrisés permettant de faire émerger la stratégie, ce qui nécessite donc de l'intégrer au processus de décision du dirigeant.

(ii) Le contrôle de gestion comme outil d'aide à la décision. L'une des défaillances du processus de prise de décision dans les structures hospitalières est la fixation cohérente des objectifs dans le temps. Ces défaillances auxquelles sont confrontés les systèmes de contrôle de gestion hospitalier peuvent être appréhendées à travers la notion de coûts cachés mise en évidence par Savall (1991) définis comme étant des dysfonctionnements générateurs de coûts non intentionnellement cachés mais invisibles car non calculés. Le contrôle de gestion en tant qu'outil d'aide à la décision doit également s'appuyer sur une structure facilitant la rencontre des erreurs, des problèmes et des solutions de façon adéquate et rapide. Ceci soulève bien la nécessité d'une amélioration des outils de gestion permettant une meilleure compréhension entre personnel médical et personnel administratif ainsi qu'une prise de décision concertée. Outre la souplesse de la structure, cela demande donc également une attitude générale associée à un respect mutuel et donc une absence de méfiance envers l'ensemble des acteurs hospitaliers qu'ils soient administratifs ou médicaux. Il apparaît dès lors que la communication des résultats issus du dialogue de gestion revêt dès lors un caractère essentiel en vue de susciter l'adhésion de tous à cette démarche gestionnaire au sein d'un dialogue de gestion partagé.

(iii) Le contrôle de gestion comme support au dialogue de gestion. Tout l'enjeu consiste donc à organiser le dialogue de gestion entre l'ensemble des acteurs susceptibles de contribuer à la définition de la stratégie et en aval pour favoriser l'atteinte des axes stratégiques ainsi définis. Le vocable de « contrôle de gestion stratégique » est significatif ; il ne désigne pas celui qui intéresse les managers officiellement en charge des choix stratégiques mais celui qui s'applique aux opérationnels en soulignant l'importance d'insuffler le souci de la stratégie aux comportements des opérationnels, en l'occurrence le personnel médical. À travers cette définition, une nouvelle problématique semble s'imposer au contrôle de gestion autour de deux besoins ; d'une part un 
besoin d'instrumentation pour atteindre des objectifs, et d'autre part un besoin de mobilisation pour agir par l'intermédiaire d'autres personnes dans la lignée des travaux d'Anthony (1988) qui montre que le «manager doit atteindre des objectifs par l'intermédiaire d'autres personnes ».

L'ensemble de ces discussions font apparaître trois caractéristiques du contrôle de gestion en tant qu'élément clé de la construction de la stratégie, outil d'aide à la décision et support au dialogue de gestion. Ces travaux font cependant l'hypothèse implicite que les situations de gestion sont bien cadrées et qu'il n'y a pas lieu de réviser les identités des concepts. Le contrôle de gestion consiste alors à appliquer les préceptes du contrôle et de l'évaluation. Les objectifs sont connus, les métiers sont stables et leurs interactions planifiées. Les méthodes sont génériques et les processus sous contrôle.

\subsection{Les fondements du contrôle de gestion à l'hôpital}

Cette hypothèse nous semble discutable dans le domaine du contrôle de gestion à l'hôpital. Celui-ci, mobilise des techniques de diagnostic, des compétences, des formes d'organisation et de coopération souvent inédites. De nouveaux objectifs apparaissent et les étapes de validation sont à compléter. Le contrôle de gestion médicalisé ne se limite pas, en effet, à un processus de conception réglée dans le cadre d'une trajectoire technologique établie (dominant design²), sous forme d'une simple adaptation du contrôle de gestion classique et des compétences existantes. Il peut impliquer un processus de conception innovante (Hatchuel, Le Masson, 2007), c'est-à-dire une révision profonde de l'identité des objets, des compétences, des raisonnements et des outils de gestion associés par rapport à des objectifs peu spécifiés au départ.

En effet, la littérature contemporaine qui traite du fonctionnement du système hospitalier se caractérise par une grande diversité des approches qu'elles soient économistes, sociologiques ou gestionnaires. Les théories économiques se sont ainsi essentiellement intéressées à la régulation externe du système hospitalier. Il faut attendre les années 1980 pour voir apparaître les premières analyses internes du fonctionnement de l'hôpital et l'entrée dans la « boite noire »à travers l'étude de la production hospitalière ; ces travaux insistent sur la complexité du produit hospitalier du fait de trajectoires-Patients singulières (Minvielle, 1996), la mesure de l'activité difficilement quantifiable (De Pouvourville, 1996 ; Moisdon et Tonneau 1999) ou encore la fonction de production et de coût de l'hôpital à travers les économies d'échelle et de gamme (De Pouvourville, 1996) ou l'inélasticité de la demande. Le recours aux théories socio-organisationnelles va permettre d'avancer dans la compréhension du fonctionnement de l'hôpital en étudiant principalement les modes de coordination interne et en intégrant l'environnement hospitalier.

Finalement, peu de travaux s'intéressent à la fonction même de contrôle de gestion à l'hôpital, alors même qu'on assiste à une prolifération d'outils de gestion au sein de cette organisation. Comment expliquer ce paradoxe ? Sans aller jusqu'à parler de contrôle de gestion, cette problématique de la faiblesse de la comptabilité de gestion à l'hôpital est commune à l'ensemble du secteur public et suscite interrogation, expérimentation, étonnement ou encore critiques tant le sujet du calcul des coûts est sensible dans une organisation censée assurer le service public alors même que ces techniques sont parfaitement balisées dans les entreprises privées. Dit autrement, peut-on et est-il souhaitable d'analyser les coûts dans les organisations publiques (Burlaud, 2005 ; Gibert, 1980).

\footnotetext{
${ }^{2}$ Un dominant design désigne un ensemble de caractéristiques qui sert de référence pour une catégorie de produits, qui donne l'identité d'un produit (Abernathy et Utterback, 1978).
} 
La comparaison des expériences conduites sur le terrain, au regard des principes fondateurs du contrôle de gestion, permet de dégager des leçons sur les difficultés de l'émergence d'un pilotage médico-économique de l'hôpital :

\section{- Des connaissances morcelées}

La compréhension des écueils du contrôle de gestion à l'hôpital peut être appréhendée de manière traditionnelle à travers le double sens du mot " contrôle »; il s'agit d'une part de "vérifier », ce que fait le contrôleur de gestion et d'autre part, d' "avoir le contrôle », ce que recherche le manager. La question qui se pose est donc de savoir si le contrôle de gestion est ce que fait le contrôleur de gestion ou la finalité du processus qu'il conduit. Or, force est de constater que le contrôle de gestion à l'hôpital ne permet pas encore de parler de maîtrise même si des efforts sont faits pour aller dans ce sens. En effet, Si l'obligation légale faite aux établissements assurant le service public hospitalier de tenir une comptabilité des dépenses engagées et une comptabilité analytique a permis de progresser dans la connaissance des coûts, il convient de souligner que cette démarche s'inscrit dans une perspective de contrôle au sens de vérification par la tutelle; la comptabilité des dépenses engagées sert au suivi et au contrôle de l'exécution budgétaire ; l'analyse de l'activité et des coûts selon les principes de la comptabilité analytique est également transmise à l'autorité administrative.

\section{- Le maintien de zones de flou}

De plus, la seule instrumentation gestionnaire des hôpitaux ne suffit pas à assurer une maîtrise des activités et donc de l'organisation. L'efficacité de l'organisation dépend moins de cette instrumentation que des mécanismes relationnels qui se construisent au sein des groupes. Or, contrairement aux enseignements de la théorie de l'agence, la préservation de zones de flou à l'hôpital peut être une condition à l'équilibre du système. Cet équilibre est lié aux imperfections des instruments de gestion en place qui laissent subsister du flou permettant de limiter les conflits. Les enjeux de pouvoir peuvent dès lors être considérés comme des facteurs d'incertitude mais aussi de stabilité en limitant l'instauration d'un climat de compétition (Moisdon et Tonneau, 1999). Cette idée a été soulignée dès les années 1970 puisque "la bienheureuse incertitude qui règne avant l'introduction de la mesure chiffrée de l'action de chacun, est considérée pour la plupart des responsables comme une source de quiétude » (Gauthier et alii, 1979). La recherche de ces zones de flou génératrices d'incertitudes s'explique également par les nombreux clivages particulièrement marqués à l'hôpital entre secteur médical et secteur administratif mais aussi entre services d'hospitalisation et services médico-techniques et enfin au sein des services administratifs entre services fonctionnels et services opérationnels.

La démarche de contrôle de gestion ne semble toujours pas ancrée dans la culture hospitalière ; le vocabulaire employé traduit d'ailleurs cet élément puisque l'obligation se résume à la tenue d'une comptabilité analytique hospitalière en vue d'une vérification par la tutelle. Le passage de la vérification à la maîtrise en lien avec le pilotage suppose donc de redonner une dimension interne au contrôle de gestion mais ne pourra se faire sans une adhésion de tous les acteurs hospitaliers enfermés jusqu'ici dans une logique de clan (Ouchi, 1979). Une certaine mise sous tension de l'organisation apparaît dès lors nécessaire pour dépasser ces barrières.

\section{- Les difficultés de la «mise sous-tension »}

Ce concept développé par Burlaud (2003) semble faire la synthèse de ce renouveau du contrôle de gestion à l'hôpital. L'objectif est ainsi de comprendre les outils de gestion dans leur contexte ce qui leur donne réellement un sens. Cette «mise sous tension » apparaît dès 
lors comme une «technologie invisible » (Berry, 1983) dans la mesure où la seule performance des machines, mesurée par des outils de gestion peut être inexploitée ou inefficace si l'organisation humaine ne permet pas d'en tirer parti.

Ce faisant, le contrôle de gestion a recours à l'instrumentation, c'est-à-dire une combinaison d'outils. Les outils les plus classiques sont les méthodes de calcul de coûts permises par la comptabilité analytique, les budgets et le reporting puis les centres de responsabilité permettant la délégation de gestion et la contractualisation interne. Or ces premiers outils font l'objet d'un certain nombre de remises en cause et plaident en faveur de nouveaux outils privilégiant un décloisonnement de la structure et la prise en compte d'indicateurs autres que financiers.

À travers ces premiers éléments de définition, nous souhaitons dépasser la vision restrictive mais largement répandue qui réduit le contrôle de gestion à une « boite à outils »; or plus qu'un ensemble d'outils et de méthodes, le contrôle de gestion est avant tout un système de régulation des comportements dans une organisation.

Les travaux de Lorino et al. (2003) consacrés au statut de l'outil résument bien les deux fonctions que peut remplir l'outil de gestion :

- La première confère à l'outil une fonction de représentation en vue de répliquer la réalité et de la simuler;

- La seconde considère l'outil de manière plus pragmatique, comme moyen d'action et d'intervention dans les organisations (Moisdon, 1998), au cœur du processus d'apprentissage (Hatchuel, Molet, 1986).

Ces travaux s'intègrent dans la lignée des réflexions dites de l'action organisée qui lie la construction de l'instrumentation avec celle de l'organisation ; élaborer c'est donc avant tout intervenir dans l'organisation.

Or la prise en compte des dynamiques organisationnelles amène tout naturellement à s'intéresser aux logiques d'acteurs et aux modes de coordination associés. Cette réflexion sur la coopération, qu'elle soit inter ou intra-organisationnelle, n'est pas spécifique à l'hôpital (Smith, Caroll et Ashford, 1995) et fait naître de nouveaux modes d'organisation centrés sur le principe de coopération entre des personnes appartenant à des métiers différents (Demeestere, 1995). Les obstacles sont alors nombreux au premier rang desquels les conflits d'intérêt ou encore l'absence d'un langage commun.

Les outils semblent dès lors avoir un rôle à jouer dans la structuration de coopérations transversales. Certains auteurs considèrent que les méthodes et outils de gestion constituent une technologie de l'organisation c'est un ensemble de procédés, de dispositifs à la fois symboliques et matériels qui rendent possible une action collective coordonnées et orientée vers un but (Bayart, 2000, Berry, 1983).

L'outil de gestion est alors considéré comme un artefact qui permet l'action organisée ; il est au service d'une action, c'est un objet fabriqué qui n'est pas qu'une simple mise en forme de l'information mais doit servir de base ou d'aide dans un raisonnement. C'est en ce sens que le contrat peut contribuer à la mise en œuvre d'une véritable action organisée. Sur la base d'un diagnostic résultant de l'agrégation de connaissances, il s'agit de formuler des objectifs et de les décliner en levier d'action; l'atteinte des objectifs peut alors être suivie au fil de l'eau sur la base d'indicateurs physiques et des mesures correctives prises en cas de déviance par rapport à ces objectifs.

Comme on l'a vu plus haut, c'est l'identité même de l'objet contrôle de gestion qui devient centrale. La reformulation de l'identité de l'objet par expansion ou par révision est 
un véritable acte de conception. Encore doit-on examiner les mécanismes qui permettent de produire les partitions expansives susceptibles de convaincre de leur originalité ou leur valeur à partir des connaissances disponibles. Dans les recherches et interventions menées, les démarches de contractualisation nous semblent constituer un ingrédient essentiel dans cet acte de conception. Nous examinons plus particulièrement en quoi la démarche de contractualisation à l'hôpital est un processus de conception nécessaire pour passer des connaissances au concept d'un contrôle de gestion médicalisé.

\subsection{Théories du raisonnement de conception. Notion de modèle génératif}

La question de la représentation du raisonnement de conception a fait l'objet de nombreuses recherches Clark (1985), Marples (1961), Alexander (1964) et Simon (1962). Ces travaux montrent que tout travail de conception consiste à assurer la cohérence entre l'objet conçu et son contexte d'utilisation. La compréhension de l'interaction entre ces deux dimensions est essentielle à toute démarche d'élaboration d'un outil de gestion. La théorie C-K (Hatchuel, 2002), s'inscrit dans cette tradition et la prolonge. Pour ces auteurs, les processus de conception de nouveaux objets reposent sur la distinction formelle entre deux espaces : l'espace des concepts (C) et l'espace des connaissances ( $\mathrm{K}$, comme knowledge). L'univers (ou espace) des connaissances est l'espace des propositions qui ont un statut logique (comptabilité analytique, analyse des coûts, groupe homogène de malades...).

L'univers des concepts est l'espace des propositions qui ne sont ni vraies, ni fausses. Appliqués à notre réflexion, nous postulons que le contrôle de gestion médical répond à cette définition et constitue donc un concept dont nous étudierons les conditions d'émergence. À ce stade, nous notons que cet univers a une structure en arborescence. La base de la théorie C-K tient dans la double expansion des connaissances et des concepts proposés (figure ci-dessous).

Figure 1 - La distinction de deux espaces dans la théorie C-K

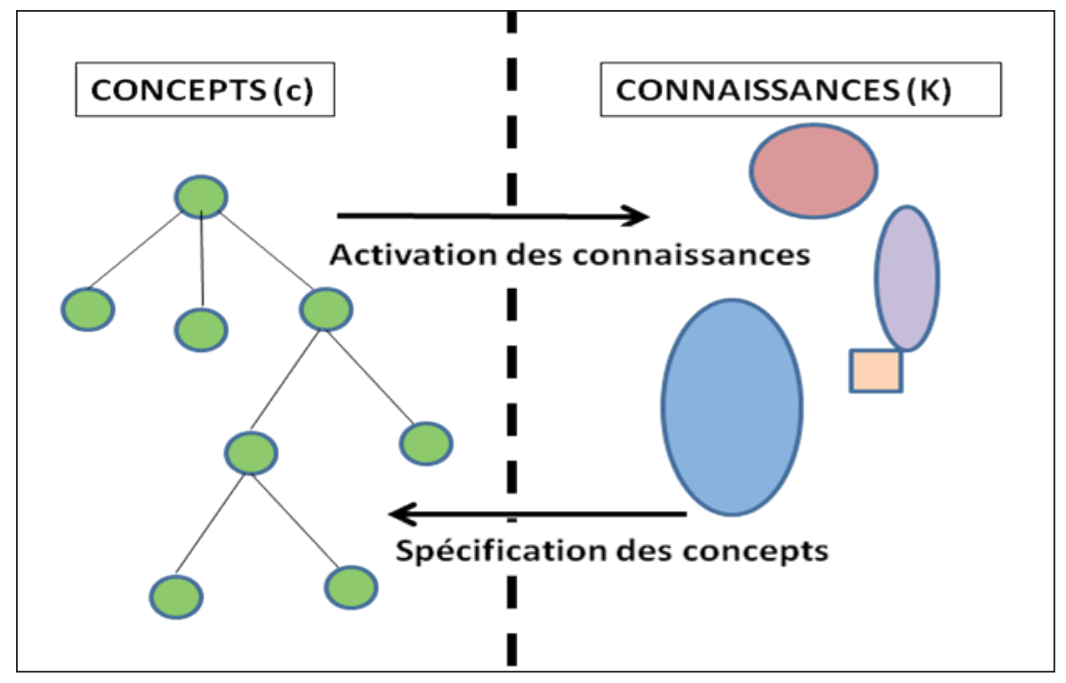


Tout en étant distincts, ces deux espaces interagissent constamment entre eux à l'aide des deux opérateurs de disjonction et conjonction, la disjonction $\mathrm{K}-\mathrm{C}$ permettant de formuler une proposition de concept à l'aide d'une connaissance alors que la conjonction C-K permet de confronter un nouveau concept à l'espace des connaissances. Seul l'espace des concepts est structuré en arbre, alors que les connaissances sont apportées de manière plus désordonnée au fur à mesure des champs d'investigations étudiés.

Cette théorie fait appel à quatre opérateurs :

- La disjonction K-C, c'est l'opération qui allant de $\mathrm{K}$ vers $\mathrm{C}$ permet la formulation d'un concept à partir d'une proposition de départ émanant de l'espace $\mathrm{K}$.

- La conjonction C-K, symétrique de la disjonction. Elle marque le moment où l'ensemble des propriétés du concept trouvé est vrai dans K. Lorsqu'une telle proposition est acceptée, le raisonnement de conception peut être arrêté.

- La déduction K-K, c'est la capitalisation des connaissances acquises qui découlent les unes des autres pour étoffer les poches de connaissances.

- La partition C-C, correspondant à la progression dans l'univers des concepts.

\section{Figure 2 - Les quatre opérateurs de la théorie C-K}

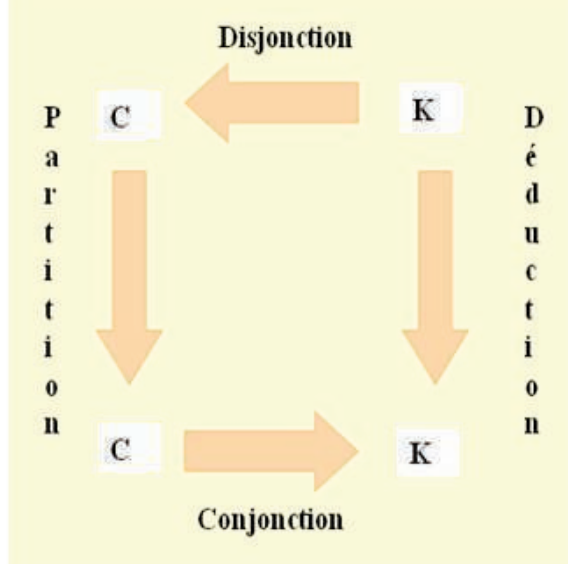

Cette représentation nous invite donc à raisonner simultanément sur deux dimensions, les concepts et les connaissances, ce que ne fait pas la littérature sur la conception de nouveaux concepts de contrôle de gestion à l'hôpital dans lesquelles les deux dimensions ne sont pas mises explicitement en relation. En s'appuyant sur la théorie C-K, c'est la disjonction qui constitue l'une des difficultés majeure à l'hôpital dans la mesure où le passage des connaissances vers de nouveaux concepts ne va pas de soi sans une réflexion sur les démarches de contractualisation. Celles-ci constituent aujourd'hui une opportunité pour redonner au contrôle de gestion à l'hôpital un sens large de pilotage médico-économique.

\subsection{Le statut du contrat de pôle}

Si l'on se focalise sur la partie décentralisée de la gestion vers les pôles, la seule existence du contrat entre direction générale de l'hôpital et les pôles médicaux ne suffit pas à assurer 
l'émergence d'un contrôle de gestion médicalisé et partagé. Cette démarche implique une circulation de l'information entre le niveau opérationnel et le niveau stratégique en vue de la fixation des objectifs et de leur éventuelle révision ainsi que le suivi des résultats au fil de l'eau à travers la conception de tableaux de bord.

La définition du contrat nous amène également à réfléchir sur son caractère synallagmatique remis en cause tant qu'aucun mécanisme de sanction ou de récompense n'est associé à ce type de contrats. En effet, on peut s'interroger sur le caractère véritablement contractuel de telles conventions. Peut-on réellement parler de contrat, défini au sens juridique comme étant une convention par laquelle une ou plusieurs parties s'obligent envers une ou plusieurs autres à faire ou à ne pas faire quelque chose ? Quelle validité accorder à un contrat pour lesquels des acteurs s'engagent à atteindre des objectifs sans maîtriser les moyens, alors que le niveau de demande donc d'activité est conditionné par les besoins de santé de la population.

\section{Le contrat comme catalyseur d'une vision partagée du contrôle de gestion médicalisé}

Il s'agit de comprendre comment le contrat organise le passage vers un nouveau concept de contrôle de gestion sur la base d'une articulation des connaissances. Notre démarche de recherche nous amène à considérer le mode de recherche et d'obtention de connaissances nouvelles comme un processus de conception.

Nous formulons ici la proposition suivante : plus que le contrat, c'est le processus de contractualisation qui permet de réunir les conditions favorables à l'émergence du concept de contrôle de gestion médicalisé et partagé. ${ }^{3}$ Pour illustrer cette discussion, nous partons de l'exemple d'un hôpital initiant une démarche de contractualisation.

\subsection{La démarche de contractualisation comme interface entre concept et connaissances}

Le point de départ de la recherche vise à explorer de nouveaux concepts de contrôle de gestion à partir d'un espace de connaissances diverses.

- des connaissances propres à l'établissement

Il s'agit de données quantitatives permettant un suivi de l'activité, de l'occupation de la structure, de la consommation et de la production d'actes supports (examens de laboratoire, examens d'imagerie, consultations, taux de passage en réanimation). Ces données quantitatives peuvent être traduites en termes monétaires et financiers dans le cadre de la Comptabilité Analytique Hospitalière (CAH) permettant ainsi de calculer le coût d'un séjour hospitalier, mais aussi les recettes procurées (tarification à l'activité pour les séjours hospitaliers, rémunération d'actes externes, enveloppes liées aux Missions d'Intérêt Général et d'Aide à la Contractualisation (MIGAC)...) et in-fine de calculer le résultat économique de l'hôpital ou d'un pôle clinique. Ces données quantitatives permettent également d'apprécier la qualité

\footnotetext{
${ }^{3}$ Cette question du partage atteste de la nécessité d'un ou plusieurs contrats permettant de coordonner et rendre compatible l'ensemble des projets médicaux au sein de l'établissement; il s'agit ainsi de dépasser la logique de construction de règles et de procédures pouvant freiner l'appropriation des projets et leur déploiement sur le terrain.
} 
des soins délivrés par l'établissement (engagement des pôles en matière d'hygiène et de prévention des infections nosocomiales, nombre de patients réadmis, nombre de patients ayant attendu plus de 30 minutes en consultation...).

Ces données ne présentent pas d'intérêt en tant que telles; elles ne constituent véritablement une connaissance qu'à partir du moment où elles sont confrontées aux mêmes données d'un panel d'établissement supposé représentatif.

\section{- des connaissances communes à plusieurs établissements}

Un établissement dispose d'un autre type de connaissances à travers les différents référentiels existants. Citons par exemple l'Échelle Nationale des Coûts Commune (ENCC) qui fournit le coût moyen décomposé d'un groupe homogène de malades (GHM) donné sur la base de la moyenne de 33 établissements. Citons également la base d'Angers qui fournit le coût moyen d'un certain nombre d'unités d'œuvre telles que le coût d'un repas, le coût d'un kg de linge, le coût d'un B (unité de mesure des examens de laboratoire)... D'autres référentiels existent en fonction du type d'établissement étudié; ainsi la base Inter CHU réunit $30 \mathrm{CHU}$ et fournit des données relatives aux durées moyennes de séjours (DMS), au Poids Moyen du Cas traité (PMCT), à la part des séjours de moins de 24 heures et permet ainsi d'apprécier la performance de son établissement par rapport à cette moyenne. Enfin des référentiels en termes de qualité des soins peuvent constituer une source d'information pertinente telle que les résultats issus de l'Évaluation des Pratiques Professionnelles ou encore de la procédure d'accréditation effectuée par la Haute Autorité de Santé (HAS).

Cette réflexion sur l'espace des connaissances soulève une question nouvelle, celle d'une corrélation entre la capacité à identifier et mesurer ces connaissances, le niveau de compétence du contrôle de gestion et l'opportunité qui en découle pour mettre en œuvre efficacement la démarche de contractualisation. Or, les hôpitaux sont caractérisés par une grande diversité en matière de contrôle de gestion, certains se contentent de répondre aux demandes de la tutelle d'autres vont plus loin en menant de véritables études médico-économiques de projets stratégiques clairement identifiés. Parmi les facteurs explicatifs on peut citer, l'existence d'une équipe spécialement dédiée au contrôle de gestion, l'expérience de leur contrôleur de gestion et sa connaissance de l'hôpital ainsi que l'accessibilité des données qu'il s'agisse de la qualité du système d'information hospitalier ou du recueil sur le terrain.

Dès lors le passage de l'espace des connaissances à celui du concept ne possède pas de frontières clairement définies. La transition vers le concept; suppose d'articuler l'ensemble de ces connaissances au sein d'un processus en trois temps. Toute exploration de nouvelles formes de contrôle de gestion solutions repose sur un changement de la structure de l'espace K des connaissances.

Illustrons ce mécanisme à partir d'un exemple simplifié ${ }^{4}$ celui d'un établissement dont les compétences en contrôle de gestion peuvent être considérées comme moyennes et qui se trouve au début son processus de contractualisation.

Étape 1 : comparaison de variables stratégiques a la moyenne de la base Inter CHU.

Cette étape a pour objectif de dégager des leviers d'actions stratégiques c'est-à-dire des segments sur lesquels des efforts devront être réalisés et feront l'objet d'un engagement. Cinq indicateurs sont étudiés :

\footnotetext{
${ }^{4}$ Les chiffres sont donnés à titre illustratif. Il s'agit d'un exemple de mise en place d'un processus de contractualisation dans un hôpital.
} 
- La Durée Moyenne de Séjours (DMS) dans la mesure où une durée trop longue génère des coûts supplémentaires notamment hôteliers ainsi que des coûts d'opportunité liés à des journées non rémunérées du fait de l'occupation des lits par ces longs séjours sans qu'aucune recette ne soit générée par des séjours supplémentaires;

- Les journées extrêmes hautes qui correspondantes à des recettes supplémentaires à ajouter aux recettes des GHS concernant les séjours dont la durée de séjour est supérieure à la borne haute;

- La part des séjours de la CMD 24 qui correspondent à des séjours courts de moins de $48 \mathrm{~h}$ répondant à une hospitalisation de jours permettant d'économiser des coûts hôteliers notamment;

- La part des « 0 jour » dans la CMD 24 qui correspond à une hospitalisation partielle;

- Le taux d'occupation des lits.

Tableau 1 - Comparaison de la moyenne de $30 \mathrm{CHU}$ avec le CHU étudié en termes de séjours, recettes et Poids Moyen du Cas Traité.

EXH : Extrêmes « hauts ", EXB : Extrêmes " bas "

\begin{tabular}{|l|l|l|r|}
\hline & CHU étudié & Base InterCHU & \multicolumn{1}{|c|}{ Rang } \\
\hline & Montant & Montant & $9^{\circ}$ \\
\hline Séjours CMD non 24 & & & $10^{\circ}$ \\
\hline Recettes des GHS & & & $21^{\circ}$ \\
\hline PMCT & & & $4^{\circ}$ \\
\hline & & & $5^{\circ}$ \\
\hline \hline Recettes EXB & & & $11^{\circ}$ \\
\hline Recettes EXH & & & $21^{\circ}$ \\
\hline PMCT & & & $26^{\circ}$ \\
\hline & & & $\mathbf{8}^{\circ}$ \\
\hline \hline Séjours CMD 24 \& séances & & & $16^{\circ}$ \\
\hline Part de CM 24 & & & \\
\hline PMCT & & & \\
\hline
\end{tabular}

Globalement, cette première analyse peut fournir un certain nombre d'informations intéressantes en termes stratégiques (tableau 1).

En termes de recettes, le Poids Moyen du Cas Traité (PMCT) des GHS de plus de 48 heures est relativement faible et place le $\mathrm{CHU}$ étudié en $21^{\mathrm{e}}$ position alors qu'il est 9éme en activité.

En termes de durée de séjours, les journées extrêmes hautes sont deux fois plus importantes avec une $5^{\mathrm{e}}$ position en termes de montant des journées extrêmes hautes. Ce nombre important de journées supplémentaires a pour corollaire une DMS plus élevée ce qui génère des coûts supplémentaires mais aussi un coût d'opportunité qui s'apparente à un nombre de journées non rémunératrices puisqu'aucun séjour supplémentaire ne correspond à ces journées. 
En termes d'activité enfin, la part des séjours de - de $48 \mathrm{~h}$ est faible et place le CHU en $21^{\mathrm{e}}$ positon.

Dès lors, deux analyses complémentaires peuvent être menées concernant la DMS et la part des séjours de « 0 séjour » au sein des séjours de - de 48h (CMD 24).

L'analyse de la DMS fait apparaître une durée supérieure tant en chirurgie qu'en médecine. L'une des explications possibles peut provenir de la part importante de journées extrêmes hautes au CHU qui témoigne d'un allongement excessif des séjours

Tableau 2 - Comparaison des DMS de la moyenne de $30 \mathrm{CHU}$ avec le CHU

\begin{tabular}{|l|c|c|c|}
\hline DMS & CHU étudié & Base Inter CHU & Écart \\
\hline Médecine & 9 & 8 & +1 \\
\hline Chirurgie & 8,5 & 8 & $+0,5$ \\
\hline
\end{tabular}

Tableau 3 - Analyse du Case Mix CMD 24

\begin{tabular}{|l|c|c|}
\hline DMS & CHU étudié & Base Inter CHU \\
\hline & Part & Part \\
\hline Séjour de 1 jour & $40 \%$ & $25 \%$ \\
\hline Séjour de 0 jour & $60 \%$ & $75 \%$ \\
\hline Totla CM24 (hors IVG) & $100 \%$ & $100 \%$ \\
\hline
\end{tabular}

Au sein de la CMD 24, la part des séjours de 1 jour est de $40 \%$ au CHU contre $25 \%$ en moyenne dans la base inter $\mathrm{CHU}$; la part des « 0 jour » est donc plus faible au sein du CHU étudié (tableau 3).

Étape 2 : simulation de l'impact en cas d'atteinte de l'objectif.

Cette étape vise à tester la pertinence des stcénarios envisagés sur la base d'une mesure d'impact en cas d'alignement sur la référence.

Le choix d'un objectif à atteindre tant en termes quantitatifs que qualitatifs suppose une prise de décision. L'un des enjeux propres au contrôle de gestion hospitalier réside donc dans l'amélioration du processus de prise de décision sur la base d'une vision prospective. Toutefois, il ne semble pas opportun de fixer un objectif qui ne procurerait pas de résultat significatif en termes financiers. La deuxième étape de la démarche de contractualisation émergente dans le CHU étudié consiste donc à simuler l'impact en termes d'activité et de recettes en cas d'alignement sur le niveau des hôpitaux les plus performants.

Diverses expériences contractuelles menées à l'hôpital fournissent une analyse précise des enjeux liés à la contractualisation. De manière schématique, deux situations extrêmes peuvent être observées dans les hôpitaux. La première est celle d'un hôpital, qui dispose d'un dispositif de contrôle de gestion précis alliant requêtes informatisées et études sur le terrain coûteuses en temps mais permettant un recueil de données adaptées à des études médico-économiques très fines. Si un tel système permet d'aider à la décision au niveau de l'établissement, assurant ainsi une circulation du niveau opérationnel vers le niveau stratégique, mais non en sens inverse, la communication des résultats étant trop limitée. Le manque de généricité entre études se traduit également par des difficultés à légitimer la démarche sur l'ensemble de l'établissement, les services cliniques n'ayant pas de vision sur les opportunités offertes par les données chiffrées. 
Tableau 4 - Étude de quelques scenarii de gestion

\begin{tabular}{|c|c|c|c|c|c|}
\hline \multirow[b]{2}{*}{ Leviers d'action } & \multirow[b]{2}{*}{ Constat } & \multirow[b]{2}{*}{ Simulation } & \multicolumn{3}{|c|}{ Gains en chirurgie et en médecine } \\
\hline & & & & Nb de séjours & Recettes \\
\hline \multirow[t]{2}{*}{ DMS } & \multirow{2}{*}{$\begin{array}{l}\text { DMS CHU >DMS } \\
\text { nationale }\end{array}$} & \multirow{2}{*}{$\begin{array}{l}\text { Application de la DMS des } \\
\mathrm{CHU} \text { au Case Mix du CHU }\end{array}$} & Chirurgie $+48 h$ & + séjours & $+\mathrm{k} \epsilon$ \\
\hline & & & Médecine $+48 h$ & & \\
\hline \multirow{2}{*}{$\begin{array}{l}\text { Journées } \\
\text { extrêmes } \\
\text { hautes }\end{array}$} & \multirow{2}{*}{$\begin{array}{l}\text { Part des journées } \\
\text { hautes } \mathrm{CHU}>\text { Part } \\
\text { nationale }\end{array}$} & \multirow{2}{*}{$\begin{array}{l}\text { Application du \% des } \mathrm{CHU} \\
\text { au Case Mix du CHU }\end{array}$} & "Chirurgie $+48 h$ & & \\
\hline & & & Médecine $+48 h$ & & \\
\hline \multirow{2}{*}{$\begin{array}{l}\text { DMS Hors } \\
\text { Extrêmes } \\
\text { hauts }\end{array}$} & \multirow{2}{*}{$\begin{array}{l}\text { DMS Hors Extrêmes } \\
\text { Haut } \mathrm{CHU}>\mathrm{DMS} \\
\text { nationale }\end{array}$} & \multirow{2}{*}{$\begin{array}{l}\text { Application de la DMS des } \\
\text { CHU au Case Mix du CHU }\end{array}$} & Chirurgie $+48 h$ & & \\
\hline & & & Médecine + 48h & & \\
\hline \multirow[t]{2}{*}{ Part CMD 24} & \multirow{2}{*}{$\begin{array}{l}\text { Part des séjours de - } \\
\text { de } 24 \mathrm{~h} \text { par rapport aux } \\
\text { séjours de - de } 48 \mathrm{~h}< \\
\text { Part des autres } \mathrm{CHU}\end{array}$} & \multirow{2}{*}{$\begin{array}{l}\text { Alignement de la part des } \\
\text { séjours de - de } 24 \mathrm{~h} \text { par } \\
\text { rapport aux séjours de - de } \\
48 \mathrm{~h} \text { sur la part des autres }\end{array}$} & Chirurgie $+48 h$ & & \\
\hline & & & Médecine $+48 h$ & & \\
\hline \multirow{2}{*}{$\begin{array}{l}\text { Part "0 jours" } \\
\text { dans } \\
\text { CMD } 24\end{array}$} & \multirow{2}{*}{\begin{tabular}{|l|} 
Part des séjours de "0 \\
jours" dans la CMD 24 \\
$<$ Part des autres CHU
\end{tabular}} & \multirow{2}{*}{$\begin{array}{l}\text { Alignement de la part des "0 } \\
\text { jours" dans la CMD } 24 \text { sur la } \\
\text { part des autres } \mathrm{CHU}\end{array}$} & Chirurgie - 24h & & \multirow{2}{*}{$\begin{array}{l}\text { pas de recettes } \\
\text { supplémentaires mais } \\
\text { des gains de places et } \\
\text { de dépense }\end{array}$} \\
\hline & & & Médecine - 24h & & \\
\hline $\begin{array}{l}\text { Taux } \\
\text { d'occupation }\end{array}$ & TO<90\% & +2 à 3 points & & & \\
\hline
\end{tabular}

À l'inverse, une démarche de dialogue de gestion véritablement institutionnalisée alliant outil et communication doit permettre l'émergence d'un contrôle de gestion médicalisé utile au décideur hospitalier dans ses choix stratégiques.

\section{Figure 3 - Démarche C-K à l'hôpital}

Etape 1 : identification des leviers d'action par la méthode du benchmarking

Connaissances internes
Données d'activité : séjours, taux d'occupation,
nombre de B de labo...
Données financières : coût séjour, recettes,
enveloppes MIGAC...
Données qualitatives : infections nosocomiales;
nb de réadmissions, temps d'attente en
consultation

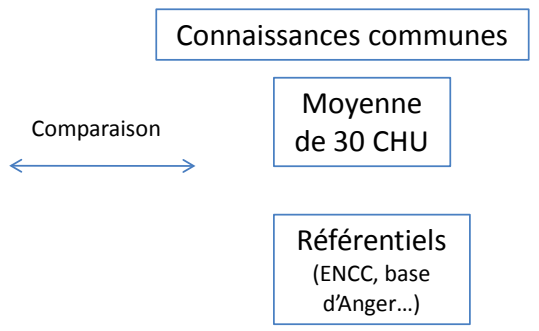

Etape 2 : test des scénarios de gestion retenus

Impact d'une réduction de la DMS

Impact d'un accroissement des séjours de la

Connaissances projetées

CM 24 (séjours de - de 48h)

Impact d'un accroissement des séjours de 0

jour au sein de la CM 24

Impact d'un meilleure occupation des lits

Etape 3 : déclinaison par pôle, fixation et suivi des objectifs via la construction d’un tableau de bord 
Étape 3 : déclinaison des leviers d'action repérés et validés au niveau de l'établissement en objectifs formulés par pôle en fonction de son activité et de ses contraintes.

Cette étape constitue alors l'output du processus de contractualisation interne; il s'agit de définir les objectifs formulés au niveau des pôles, formaliser des indicateurs adaptés pour permettre un suivi de l'atteinte de ces objectifs, proposer des mesures correctives en cas d'écarts importants et ainsi mettre en œuvre un véritable pilotage au fil de l'eau.

L'ensemble de cette démarche a priori séduisante semble toutefois atteindre plusieurs limites.

- La question de la pertinence et de la fiabilité des données

La première limite est générale, elle concerne la critique des référentiels utilisés tels que l'ENCC ou la base d'Angers dont la méthodologie peut être discutée et qui ne permet que d'obtenir des moyennes de données observées; s'agissant d'une démarche de benchmarking, on peut d'ailleurs s'interroger sur la pertinence d'une comparaison à une moyenne et non aux meilleures pratiques.

Par ailleurs, les données en internes doivent faire l'objet d'un travail de vérification afin de s'assurer de la fiabilité du contrôle de gestion; dans le cas inverse, le risque consisterait à ancrer des décisions sur un diagnostic erroné. Cet écueil peut d'ailleurs être l'une des explications au gap persistant entre gestion et organisation.

\section{- Le gap persistant entre gestion et organisation}

Notre étude confirme en effet un des reproches majeurs fait au contrôle de gestion hospitalier, celui d'être cantonné à la seule logique budgétaire; les outils sont alors confinés aux mains des seuls gestionnaires sans appropriations par l'ensemble des parties prenantes. En effet, notre exemple montre bien l'importance accordée au diagnostic, or il s'agit d'un diagnostic basé sur des données d'activité lesquelles sont difficile à manipuler tant les classifications se révèlent complexes. Le risque est alors de restreindre le contrôle de gestion à l'élaboration du diagnostic; au mieux cette démarche permettrait de vérifier la viabilité économique de la démarche stratégique sans pour autant valider l'acceptabilité fonctionnelle et organisationnelle des hypothèses de travail auprès des équipes médicales et soignantes.

- La difficulté de l'appropriation d'une démarche gestionnaire par les équipes terrains

Outre la complexité des données à manipuler en amont, le contrôle de gestion souffre d'une difficulté liée à la présentation des résultats ; quel niveau de détail doit-on retenir pour communiquer les résultats de notre étude aux pôles et sur cette base discuter des projets médicaux? Plus on décline et on descend dans le niveau de détail plus on est confronté à la multiplicité des éléments du diagnostic à analyser mais dès que l'on remonte, on court le risque d'occulter certains éléments du diagnostic.

Enfin, la seule mise en œuvre d'indicateurs quantitatifs ne peut pas être considérée comme le seul élément de la démarche de contrôle de gestion dans la mesure où le corps médical est avant tout sensible à des indicateurs médicaux dont les effets directs sur le patient semblent évidents. Dès lors, ces tableaux de bord de suivi budgétaires doivent être complétés par des indicateurs de suivi de la qualité intégrés au sein du contrat de pôles, garants de l'acceptabilité des équipes médicales et soignantes à la démarche gestionnaire (Gibert, 2009). 


\subsection{La contractualisation comme processus de révision du contrôle de gestion à l'hôpi- tal et de spécification d'un pilotage médico-économique}

De manière plus conceptuelle, les difficultés rencontrées illustrent la nécessité d'une démarche de contractualisation qui organiserait l'articulation des connaissances en vue de s'engager sur des objectifs pertinents et réalistes. Ainsi, la restructuration de l'espace $\mathrm{K}$ apparaît nécessaire à l'extension de $\mathrm{K}$ au-delà de ses limites (liens entre différentes poches de connaissances, émergence de nouvelles poches indispensables à la progression et à l'extension du contrôle de gestion classique). Cette interaction dans l'espace $\mathrm{K}$ va permettre de remonter d'un contrôle de gestion classique « confiné » vers un contrôle de gestion innovant à l'hôpital et d'opérer ainsi une disjonction dans l'espace des concepts.

Le processus de contractualisation s'apparente donc à un processus de conception qui vise d'abord à restructurer l'espace des connaissances et organiser ensuite le passage d'un raisonnement de l'échelle macroéconomique à la mise en œuvre davantage microéconomique. C'est l'ensemble de ce processus qui permet de mettre en œuvre un véritable dialogue de gestion médicalisé et partagé et constitue une démarche complète.

Figure 4 - Processus de contractualisation et structuration de l'espace de connaissances

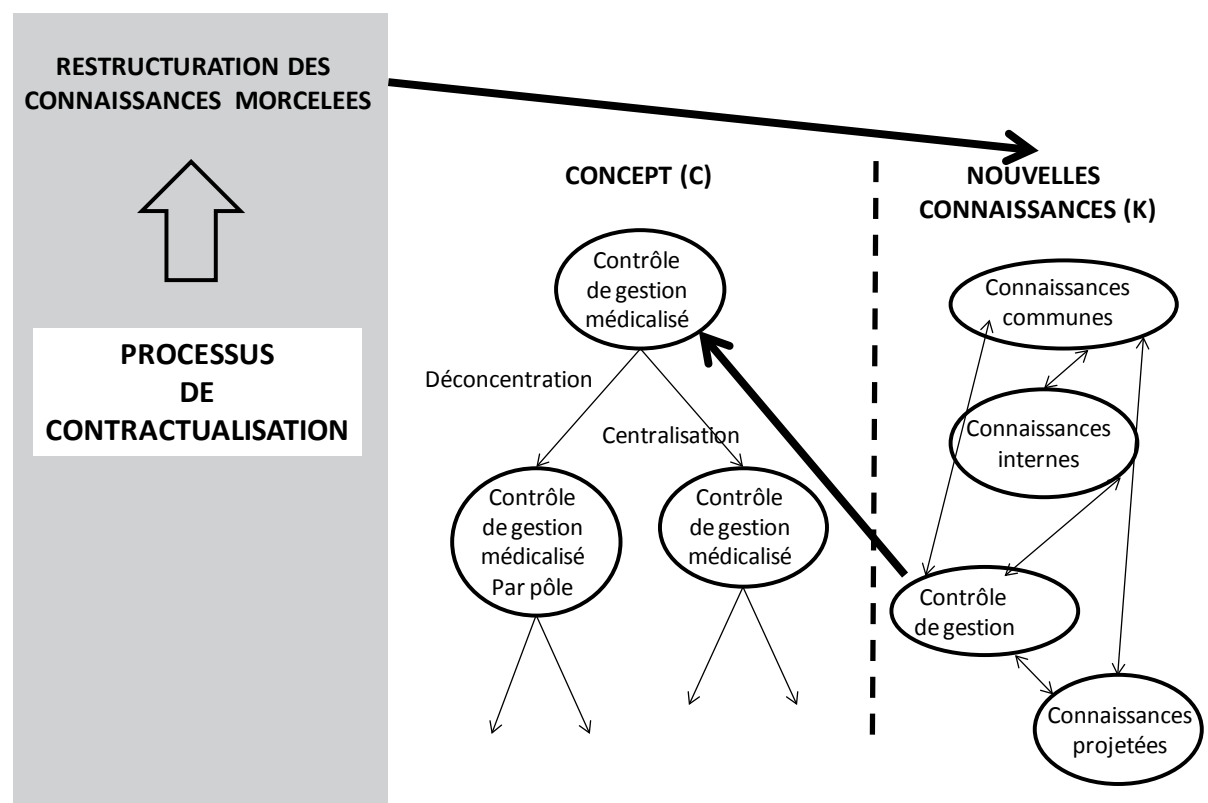

Le contrôle de gestion médicalisé et partagé est scindé en deux ; une partie déconcentrée vers les pôles, sur la base d'un transfert des compétences vers le cadre administratif de pôle, également appelé assistant de gestion, dont les missions telles que l'analyse et le suivi de tableaux de bord médico-économiques, la formalisation de propositions d'optimisation des recettes du pôle s'apparentent à des missions de contrôle de gestion. Une partie centralisée sous la responsabilité de la cellule Contrôle de gestion centralisée ${ }^{5}$ au sein des équipes de

${ }^{5}$ Rattachement à la direction des finances ou à la direction générale. 
direction et qui concerne la synthèse des résultats et la production des documents tels que les Comptes de Résultats Analytique (CREA), les Tableaux Coût Case Mix (TCCM).

L'apport en connaissances ne réside pas tant dans les outils mis en œuvre, il ne s'agit que de tableaux de bords, mais dans le processus de contractualisation interne dans son ensemble. Les projets de contractualisation - déconcentration de la gestion au sein des pôles mobilisent plusieurs facettes du management et de la conduite de projets.

- Le contrat apparaît comme un instrument permettant d'accroître la contribution des pôles à la gestion. Outre la dimension stratégique à travers la production du projet de pôle, c'est en termes d'organisation que la contribution des pôles est attendue. Notre expérience montre que l'un des enjeux consiste à repenser la prise en charge globale des patients sur la base d'une harmonisation des protocoles médicaux et soignants et donc du poids relatifs des soins externes, de l'hospitalisation de jour, de semaine ou traditionnelle.

- La phase de construction des contrats participe à la mise en œuvre d'un nouveau système de pilotage. Le « rendre compte », activité courante des cadres de santé, devient de plus ne plus fréquent pour les médecins. Au-delà des contenus et des rubriques de chaque tableau de bord, c'est le travail de conception des tableaux de bord qui devient stratégique, permettant de stabiliser les représentations de chacun (direction - médecins) autour d'un même indicateur et donc d'une connaissance nouvelle car partagée.

- La conception des tableaux de bord rend nécessaire l'apprentissage de nouveaux rôles et la compréhension des contraintes liées à l'exercice professionnel de chacun. Les tableaux de bords ainsi créés, malgré toutes les limites qu'ils peuvent présenter, agissent bien comme des technologies invisibles (Berry, 1983) à travers des échanges sur des données de gestion même incomplètes ce qui peut être considéré comme une progression de la pensée gestionnaire. En effet, tout l'enjeu consiste à faire accepter cette incomplétude au corps médical qui souhaiterait saisir toute la complexité du processus de production de soins dans les tableaux de bord.

Doit-on pour autant bannir le contrat de pôle ? Plus que le contrat de pôle c'est la démarche de contractualisation qui doit être étudiée sans se restreindre à la finalité que constitue le contrat de pôle. Affirmer que la construction du contrat de pôle, sans se focaliser sur l'outil lui-même, permet un dialogue de gestion médicalisé et partagé et considérer qu'une telle proposition est vraie, ou fausse, reviendrait, à l'inverse, à énoncer une connaissance qui arrêterait le raisonnement de conception. Le concept doit alors être reformulé en le différenciant de ce qui existe. Ceci nous amène donc à préciser que le dialogue de gestion médicalisé et partagé dans le cadre d'une démarche de contractualisation apparaît comme un vecteur permettant l'agrégation des connaissances au service de l'émergence d'un concept qui reste à préciser.

Une fois le contrat établi, la démarche de contractualisation et le processus de conception sous-jacent ne s'arrêtent pas pour autant Nakhla (2001 et 2003). L'étape de diffusion et de suivi du contrat constitue une étape à part entière en faveur d'un contrôle de gestion médicalisé et partagé. Dès lors, les, tableaux de bord n'ont de sens que s'ils permettent aux différentes parties prenantes d'échanger entre elles; les données financières sont à ce titre intéressantes mais elles seront d'autant plus commentées, critiquées et feront l'objet de propositions si elles sont visibles pour l'ensemble des acteurs hospitaliers. Certains établissements ont ainsi recours à un logiciel de cartographie pour mettre en valeur les résultats chiffrés. De cet échange peut résulter un certain nombre d'engagements faisant l'objet du contrat de pôle par exemple. 
Les nouvelles modalités d'affectation et de désaffectation des Praticiens Hospitaliers après avis de la Commission Médicale d'Établissement (CME) peuvent être interprétées comme un moyen d'aller au bout de la démarche de conception d'un contrôle de gestion médicalisé et partagé. En distinguant la responsabilité médicale du chef de service de celle du praticien responsable d'un pôle d'activité clinique ou médico-technique qui bénéficie d'une autorité fonctionnelle sur l'ensemble des équipes médicales soignantes et d'encadrement, le droit reconnaît la nécessité d'insuffler le souci d'une démarche de contrôle de gestion et de l'impliquer dans cette démarche. Cette autorité lui permet en effet de mettre en œuvre au sein du pôle la politique générale de l'établissement et les moyens correspondants aux objectifs assignés au pôle tout en construisant le projet de pôle comportant obligatoirement un volet relatif à la qualité et à l'évaluation des soins.

La contractualisation en tant qu'engagement à atteindre des résultats implique alors une démarche d'évaluation laquelle peut servir de base à l'instauration d'un mécanisme d'intéressement positif ou négatif qui apparaît comme étant l'un des moyens incitatifs les plus efficaces en vue de faire participer les pôles à ce nouveau contrôle de gestion (Nakhla, 2010).

\section{Conclusion}

L'hypothèse que nous avons formulée consiste à considérer la démarche de contractualisation comme étant l'un des fondements d'une nouvelle démarche de contrôle de gestion à l'hôpital qui tend vers le pilotage médico-économique. Ce nouveau concept doit permettre d'insuffler une certaine culture managériale à l'hôpital et illustre ainsi le passage du contrôle de gestion dans son ensemble du statut de technique dominée par les outils à celui d'une discipline tournée vers le management. Pour autant, notre expérience révèle que le contrôle de gestion même issu d'une démarche de contractualisation souffre encore d'un certain nombre d'écueils; en effet, présentée comme étant un outil à portée stratégique et organisationnelle elle n'est rien d'autre qu'un outil d'allocation des ressources certes à visée prospective mais qui demeure centré sur la modélisation de décisions stratégiques économiquement compatibles dans le cadre de préoccupations financières liées à la T2A.

L'identification du poste de contrôleur de gestion comme métier émergent et sensible par l'observatoire national des emplois et des métiers de la fonction publique hospitalière en 2007, et l'élaboration d'une fiche descriptive spécifique au répertoire des métiers de la fonction publique hospitalière atteste de la volonté de renforcer ce concept de contrôle de gestion médicalisé et partagé avec pour ambition finale le passage du statut de concept à celui de connaissance mettant ainsi fin au processus de conception. Le processus de contractualisation contribue à l'exploration et la convergence vers de nouveaux outils de pilotage spécifiques qui doivent dépasser la seule logique budgétaire et l'allocation des ressources au profit d'une analyse des processus et donc d'une modification des comportements organisationnels. C'est à cette condition que le gap existant entre contrôle de gestion et organisation pourra se réduire. 


\section{Bibliographie}

ABERNATHY W.J. et UTTERBACK J.M., (1978), Patterns of Innovation in Technology, Technology Review, 40-47.

ALEXANDER C., (1964), Notes on the synthesis of form, Harvard University Press, Cambridge, MA.

ALVAREZ F., (2000), Le contrôle de gestion en milieu hospitalier : une réponse à l'émergence des risques organisationnels, Actes du XXI Congrès de l'Association Française de Comptabilité.

ANTHONY R. N., (1988), The management control function, Harvard Business School Press.

BAYART D., (2000), Discussion : la gestion productrice de sens ?, Les Cahiers du Centre de Recherches Historique 25.

BERRY M. , (1983), Technologie invisible, Presses École Polytechnique, Paris.

BOUINOT C, (2005), Contrôle de gestion à l'hôpital, régulation et dynamique instrumentale, Thèse école des mines de Paris.

BOUQUIN H., (1997), Les fondements du contrôle de gestion, PUF, Paris.

BURLAUD A., SIMON C., (2003), Comptabilité de gestion : coûts/contrôle, Vuibert, Paris.

CLARK KB, (1985), The interaction of Design hierarchies and market concepts in the technological evolution, Research Policy 14, 235-251.

COLASSE S., (2008), Objet nouveau du contrôle de gestion à l'hôpital : entre passé, présent et futur, Communication Séminaire Défis des organisations de santé, défis des sciences de gestion - IFROSS - Lyon - novembre 2008.

DEMEESTERE R., (1995), La transversalité : gestion des processus et des projets, Revue Française de Gestion 104, 52-54.

DEMEESTERE R., LORINO P., MOTTIS N., (2003), Contrôle de gestion et pilotage, Dunod, Paris.

DE POUVOURVILLE G.,(1996), L'évaluation économique des actions des soins : modélisation analyse coûtefficacité, Sciences sociales et santé 14 (4), 65-72.

ESCAFFRE J. P., (2008), Le contrôle de gestion des unités de soins hospitaliers, Economica, Paris.

EXPERT P., (2006), La tarification à l'activité. Médecine et gestion : une dynamique nouvelle, Berger-Levrault, Paris.

GAUTHIER P. H., GRENON A., PINSON G., RAMEAU E.,(1979), Le contrôle de gestion à l'hôpital, Dunod, Paris .

GIBERT P., (1980), Le contrôle de gestion des organisations publiques, Éditions d'organisation, Paris.

GIBERT P., (2009), Tableaux de bord pour les organisation publiques, Dunod, Paris.

HATCHUEL A., MOLET H., (1986), Rational modeling in understanding and aiding human decision making, European Journal of Operational Research 24.

HATCHUEL A., (2002), La théorie $C$ - $K$ : fondements et usages d'une théorie unifiée de la conception, Colloque «Sciences de la conception », Lyon, 15-16 mars 2002.

HATCHUEL A., LE MASSON P., (2007), Les processus d'innovation, Hermès - Lavoisier, Paris.

KAPLAN R.S., NORTON D.P., (2003), Le tableau de bord prospectif, Éditions d'Organisation, Paris.

MARPLES David L., (1961), The decision of engineering design , Transactions on Engineering Management June, 55-71.

MEVELLEC P., (2004), Du projet stratégique à l'action » : la délicate configuration des outils $\mathrm{ABC} / \mathrm{ABM}$, Finance Contrôle Stratégie7 (3), 5-30.

MINTZBERG H., (1979), Structure et dynamique des organisations, Éditions d'Organisation, Paris.

MINVIELLE E, (1996), Gérer la singularité à grande échelle, Revue Française de gestion 109, 114-125.

MOISDON J. C., (1998), Du mode d'existence des outils de gestion, Seli Arslan, Paris.

MOISDON J. C., TONNEAU D., (1999), La démarche gestionnaire à l'hôpital. 1. Recherches sur la gestion interne », Seli Arslan, Paris. 
NAKHLA M., (2001), Dynamique des contrats et innovation dans l'entreprise - Évolution des modes de coordination et régulations économiques des projets, Gérer et comprendre 66.

NAKHLA M., (2003), Information, coordination and contractual relation in firms, International Review of Law and Economics 23 (1).

NAKHLA M., (2010), Designing physician's incentive for increased activity: paying for

Performance, Journal d'Économie Médicale 28 (3-4).

SAVALL H., ZARDET V., (1991), Maîtriser les coûts et les performances cachés. Le contrat d'activité périodiquement négociable, Economica, Paris.

OUCHI W. G., (1979), A conceptual framework for the design of organizational control mechanisms, Management Science (September), 833-848.

SIMON H., (1962), The architecture of complexity, Proceedings of the American Philosophical Society 106, 467-482.

SMITH K.G., CARROLL S.J., et ASHFORD S.J., (1995), Intra- and inter-organizational cooperation: Toward a research agenda, Academy of Management Journal 39, 7-23.

\section{Annexe - Méthodologie de la recherche}

La recherche menée s'appuie sur une expérimentation menée au sein d'un grand CHU de province lors de la phase initiale de conception des contrats de pôle et des tableaux de bord associés. L'étude de cas ici présentée est l'occasion d'observer de près les interactions entre connaissances médico-économiques et identifier les moments clés pour organiser des connaissances spécifiques.

Ce travail a été mené au sein d'un groupe de travail constitué de médecins et du contrôleur de gestion et, complété par des entretiens ciblés à partir des différentes rubriques du contrat (objectifs, indicateurs de suivi, révision des objectifs...). L'ensemble du processus de contractualisation observé peut être décomposé en plusieurs étapes : l'élaboration d'un diagnostic, la fixation d'objectifs, le déploiement via un plan d'action, le suivi au fil de l'eau, l'évaluation finale et la mise en place de mécanismes de sanctions récompenses.

Notre exploration a porté sur les trois premières étapes; faute de recul suffisant nous n'avons pu mesurer que les résultats au fil de l'eau, les ajustements susceptibles d'en découler et donc l'évaluation finale du niveau d'atteinte des objectifs.

L'originalité du travail mené par les cellules « Contrôle de gestion» des établissements étudiés repose sur la mise en œuvre d'une démarche de benchmarking qui vise à comparer la situation de la structure étudiée aux meilleures pratiques. Il s'agit en quelque sorte d'organiser le passage d'un raisonnement à l'échelle macroéconomique à la mise en œuvre davantage microéconomique. Le choix a été fait de s'appuyer sur des données agrégées au niveau national puis de les comparer aux performances des établissements étudiés afin de déterminer des leviers d'action stratégiques pour les établissements qui seront ensuite déclinés en interne au sein de chaque pôle.

Dans cette perspective, la démarche entreprise s'est déroulée en trois temps : l'élaboration d'un diagnostic et l'identification des leviers d'actions stratégiques propres aux spécificités des hôpitaux considérés, simulation du gain attendu et fixation des objectifs. 


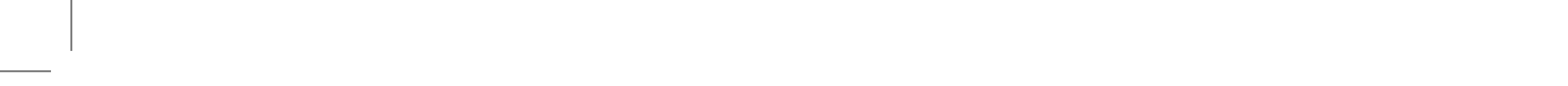

